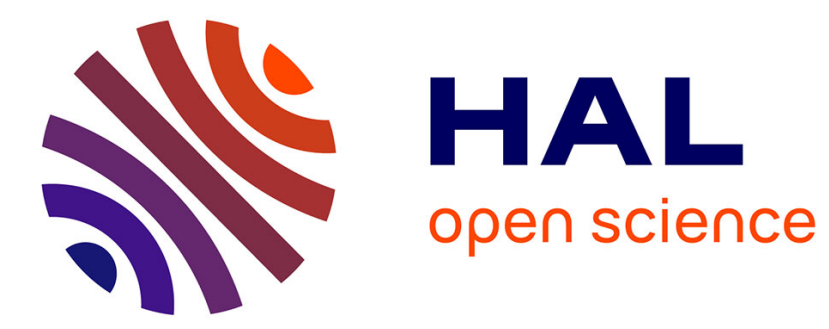

\title{
Carleman estimates for the heat equation with discontinuous diffusion coefficients
}

\author{
Olivier Poisson
}

\section{To cite this version:}

Olivier Poisson. Carleman estimates for the heat equation with discontinuous diffusion coefficients. 2008. hal-01255483

\author{
HAL Id: hal-01255483 \\ https://hal.science/hal-01255483
}

Preprint submitted on 13 Jan 2016

HAL is a multi-disciplinary open access archive for the deposit and dissemination of scientific research documents, whether they are published or not. The documents may come from teaching and research institutions in France or abroad, or from public or private research centers.
L'archive ouverte pluridisciplinaire HAL, est destinée au dépôt et à la diffusion de documents scientifiques de niveau recherche, publiés ou non, émanant des établissements d'enseignement et de recherche français ou étrangers, des laboratoires publics ou privés. 


\title{
Carleman estimates for the heat equation with discontinuous diffusion coefficients
}

\author{
Olivier Poisson \\ Laboratoire d'analyse, topologie, probabilités, CNRS UMR 6632 \\ Université de Provence, Marseille, France \\ E-mail: poisson@latp.univ-mrs.fr
}

\begin{abstract}
We consider the heat equation with a diffusion coefficient that is discontinuous at an interface. We give global Carleman estimates for solutions of this equation, even if the jump of the coefficient across the interface has not a constant sign.
\end{abstract}

AMS classification scheme numbers: 35K05, 35K55, 35R05, 35R30

\section{Introduction}

\subsection{The heat Equation with a discontinuous diffusion coefficient}

Let $\Omega \subset \mathbb{R}^{n}$ be a bounded connected open set with boundary $\Gamma$ of class $\mathcal{C}^{2}$. Let $a(\cdot)$ be a measurable function of the variable $x \in \Omega$ and satisfying

Assumption 1.1 There exist two constants $a_{\min }$, $a_{\max }$ so that $0<a_{\min } \leq a(x) \leq a_{\max }<\infty$.

We denote by $\mathcal{A}:=-\operatorname{div}(a \nabla \cdot)$ the formal stationary differential operator on $\Omega$. We consider for a time $T>0$ the formal heat operator $\mathcal{L}:=\partial_{t}+\mathcal{A}$ in the cylinder $Q=(0, T) \times \Omega$ and we define $L$ as the heat operator $L=\mathcal{L}$ with domain

$$
D(L):=\left\{q: q \in L^{2}\left(0, T ; H_{0}^{1}(\Omega)\right), \partial_{t} q \in L^{2}\left(0, T ; H^{-1}(\Omega)\right), \mathcal{L} q \in L^{2}(Q)\right\} .
$$

For $q_{0} \in L^{2}(\Omega)$ we consider the following equations:

$$
\left\{\begin{array}{l}
\mathcal{L} q=f \text { in } Q, \\
q(0, \cdot)=q_{0} \text { in } \Omega .
\end{array}\right.
$$

The function $q$ then satisfies the Dirichlet homogeneous condition $q(t, x)=0$ for $(t, x) \in \Sigma:=(0, T) \times \Gamma$. We know that if $f \in L^{2}\left(t_{0}, T ; H^{-1}(\Omega)\right)$ then (1.1) admits an unique solution we denote by $Y\left(f, q_{0}\right) \in L^{2}\left(0, T ; H_{0}^{1}(\Omega)\right)$, and if $f \in L^{2}(Q)$ then $Y\left(f, q_{0}\right) \in D(L)$. (We refer to [1, Theorems 1.1, 4.1 and Remark 4.3; Chap. 3] and to [2] for a complete proof). We make the following assumptions on the diffusion coefficients $a(\cdot): a(x)$ is piecewise smooth in $\Omega$; more precisely, $\Omega$ admits a partition in two non 
empty open sets, $\Omega_{0}$ and $\Omega_{1}=\Omega \backslash \overline{\Omega_{0}}$, such that $a_{j} \equiv a_{\mid \Omega_{j}} \in \mathcal{C}^{1}\left(\overline{\Omega_{j}}\right), j=0,1$. Moreover the interface $S=\overline{\Omega_{0}} \cap \overline{\Omega_{1}}$ is of class $\mathcal{C}^{2}$. We denote by $n$ the outward unit normal to $\Omega_{1}$ at the points of $S$ and also the outward unit normal to $\Omega$ at the points of $\Gamma$. Formally, the jump of a quantity $q$ on $S$ is defined by $[q]:=q_{0}-q_{1} \equiv\left(q_{\mid \Omega_{0}}\right)_{\mid S}-\left(q_{\mid \Omega_{1}}\right)_{\mid S}$. So $S$ is partitioned in $S=S^{+} \cup S^{-} \cup S^{0}$ with

$$
S^{ \pm}:=\{x \in S ; \pm[a](x)>0\}, S^{0}=\{x \in S ;[a](x)=0\} .
$$

We shall analyse (1.1) in the large framework $S \neq S^{0}$, that is, when $a$ is discontinuous across $S^{+} \cup S^{-} \neq \emptyset$.

We look for global Carleman estimates on $Y\left(f, q_{0}\right)$ with source terms in $\omega_{T}=(0, T) \times \omega$, where $\omega \subset \Omega$ is open, non empty, and satisfies

Assumption 1.2 Each connected component of $\Omega_{0}$ contains part of $\omega$.

Then there are two different situations that will be analysed in this work.

- Case 1: $\Omega_{0} \subset \subset \Omega$ (see Fig. 1);

- Case 2: $\Omega_{1} \subset \subset \Omega$ (see Fig. 2).

The main goal of this paper is to obtain a Carleman estimate for q solution of (1.1) in cases 1 or 2.

\section{Main Results}

In order to state the main result of this work, we need some geometrical conditions (see Figure 2).

\subsection{Geometrical Conditions and Technical Assumptions}

Assumption 2.1 on $\left(\Omega_{1}, S\right)$; corresponding to case 1. There exists a vector field $\zeta$ : $\overline{\Omega_{1}} \rightarrow \mathbb{R}^{n}, \zeta \in \mathcal{C}^{1}\left(\overline{\Omega_{1}}\right)$, so that

$$
\begin{aligned}
& \zeta(x) \cdot n(x)<0 \quad \forall x \in \Gamma, \\
& \zeta(x) \cdot n(x)>0 \quad \forall x \in S, \\
& \zeta(x) \neq 0 \quad \forall x \in \overline{\Omega_{1}},
\end{aligned}
$$

and if we consider the characteristics associated to $\zeta$

$$
\left\{\begin{array}{l}
\frac{d x(t)}{d t}=\zeta(x(t)), t>0, \\
x(0)=x_{0}
\end{array}\right.
$$

with $x_{0} \in \Gamma$, we also assume that for some time $T_{1}>0$ and for every $x_{0} \in \Gamma$, there exists $t_{1}\left(x_{0}\right)<T_{1}$ such that the solution $x(t)$ of (2.1) verifies

$$
x(t) \in \Omega_{1} \text { for } 0<t<t_{1}\left(x_{0}\right) \text {, and } x\left(t_{1}\left(x_{0}\right)\right) \in S .
$$

See figure 1 and [3, page 624]. 
Assumption 2.2 on $\left(\Omega_{1}, S\right)$; corresponding to case 2. There exists an open set $\mathcal{O} \subset \subset \Omega_{1}$ (with always a unit outward normal $n$ ) and a vector field $\zeta: \overline{\Omega_{1}} \rightarrow \mathbb{R}^{n}$, $\zeta \in \mathcal{C}^{1}\left(\overline{\Omega_{1}}\right)$, so that

$$
\begin{array}{ll}
\zeta(x) \cdot n(x)>0 & \forall x \in S, \\
\zeta(x) \cdot n(x)>0 & \forall x \in \mathcal{O}, \\
\zeta(x) \neq 0 & \forall x \in \overline{\Omega_{1}} \backslash \mathcal{O},
\end{array}
$$

and for the characteristics associated to $\zeta$

$$
\left\{\begin{array}{l}
\frac{d x(t)}{d t}=-\zeta(x(t)), t>0, \\
x(0)=x_{0}
\end{array}\right.
$$

with $x_{0} \in S$, we also assume that for some time $T_{2}>0$ and for every $x_{0} \in S$, there exists $t_{2}\left(x_{0}\right)<T_{2}$ such that the solution $x(t)$ of (2.3) verifies

$$
x(t) \in \Omega_{1} \backslash \mathcal{O} \text { for } 0<t<t_{2}\left(x_{0}\right) \text {, and } x\left(t_{2}\left(x_{0}\right)\right) \in \partial \mathcal{O} .
$$

See figure 2 and [3, page 625].

The main restriction of this framework lies in

Assumption 2.3 The set $S^{+}$can be extended to a variety $S^{\prime}$ of class $C^{2}$ which is the interface between two open sets $\Omega_{0}^{\prime}, \Omega_{1}^{\prime} \subset \Omega$, with $\Omega_{1}^{\prime} \subset \Omega_{0}, \Omega_{1} \subset \Omega_{0}^{\prime}$ and each connected component of $\Omega_{0}^{\prime}=\Omega \backslash \overline{\Omega_{1}^{\prime}}$ contains part of $\omega$. See figures 1 and 2 .

Remark 2.1 If $S^{+}=\emptyset$ then assumption 2.3 holds for the case 1 with $S^{\prime}=\emptyset$ and $\Omega_{1}^{\prime}=\emptyset$, and for the case 2 with $S^{\prime}=\Gamma, \Omega_{1}^{\prime}=\emptyset$.

We shall need the technical assumption 2.4 below. We denote by $\nabla^{\prime}$ the tangential derivative on $S$ and we set $S^{00}:=\overline{S^{+}} \cap \overline{S^{-}} \subset S^{0}$.

Assumption 2.4 There exist a neighbourhood $V \subset S$ of $S^{00}$ and a function $h_{0} \in \mathcal{C}^{2}(V)$ such that $\left(\nabla^{\prime} h_{0}(x) \neq 0, \forall x \in V\right)$, and $\left( \pm h_{0}(x)>0, \forall x \in V \cap S^{ \pm}\right)$.

Equivalently, $S^{00}$ is a $(n-2)$-dimensional submanifold of $S$ separating $S^{-} \cap V=$ $h_{0}^{-1}\left(\mathbb{R}^{-*}\right)$ and $S^{+} \cap V=h_{0}^{-1}\left(\mathbb{R}^{+*}\right)$. Assumption 2.4 is checked if $[a(\cdot)]$ is of class $C^{2}$ in a neighbourhood of $S^{00}$ and if $\nabla^{\prime}[a(x)] \neq 0$ for any $x \in S^{00}$ : in such a case we can choose $h_{0}(x)=[a(x)]$.

Remark 2.2 If $S^{+}=\emptyset$ then $S^{00}=\emptyset$ and so assumption 2.4 is validated. However it possibly happens that sets $S^{ \pm}$are non empty but $S^{00}=\emptyset$. In such a case $\nabla^{\prime}[a]=0$ on $S^{0}=\partial S^{+} \cup \partial S^{-}$.

Remark 2.3 In assumption 2.4 the function $h_{0}$ can be extended to a function $\tilde{h}_{0} \in$ $\mathcal{C}^{2}(W)$, where $W$ is some neighbourhood of $S^{00}$ in $\Omega$, and such that the vector field $\nabla^{\prime} h_{0} \in\left(\mathcal{C}^{1}(V)\right)^{n-1}$ extends to the vector field $\nabla \tilde{h}_{0} \in\left(\mathcal{C}^{1}(W)\right)^{n}$ satisfying $\nabla \tilde{h}_{0}(x) \neq 0$ for all $x \in W$. The set $\tilde{h}_{0}^{-1}(\{0\}) \subset W$ separates $W$ into $W^{+}=\tilde{h}_{0}^{-1}\left(\mathbb{R}^{+*}\right)$ and $W^{-}=\tilde{h}_{0}^{-1}\left(\mathbb{R}^{+*}\right)$. Thus if $S^{\prime}$ is a $\mathcal{C}^{2}$ extension of $S^{+}$, then $S^{\prime} \cap W^{+}=S^{+} \cap W=S^{+} \cap V$, $S \cap W^{-}=S^{-} \cap W=S^{-} \cap V, \tilde{h}_{0}(x)<0$ for all $x \in\left(S^{\prime} \backslash \overline{S^{+}}\right) \cap W$ and $\tilde{h}_{0}(x)>0$ for all $x \in S^{+} \cap W$.

Furthermore we can choose $V, W$ sufficiently small so that $\left|\tilde{h}_{0}\right| \leq \frac{1}{2}$ in $W$. 
a)

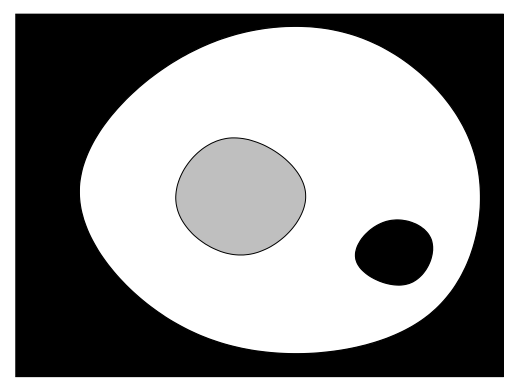

b)

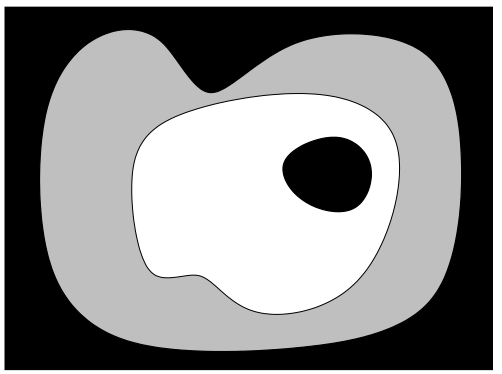

Figure 1. Case 1: Assumption 2.1 is not fulfilled in situation a). Situation in b) shows a third case.
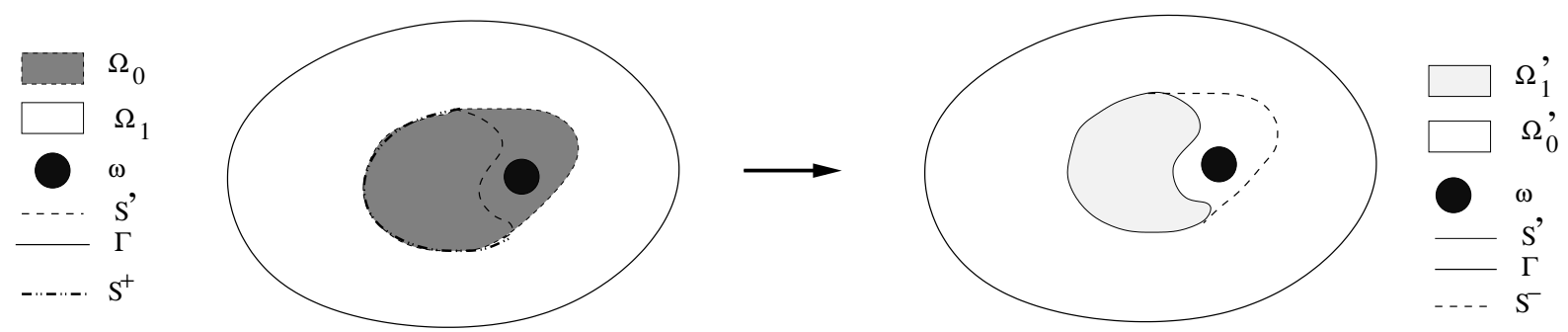

Figure 2. Case 1: Assumptions 2.1, 1.2 and 2.3 are fulfilled The arrow means that we extend $S^{+}$to $S^{\prime}$.
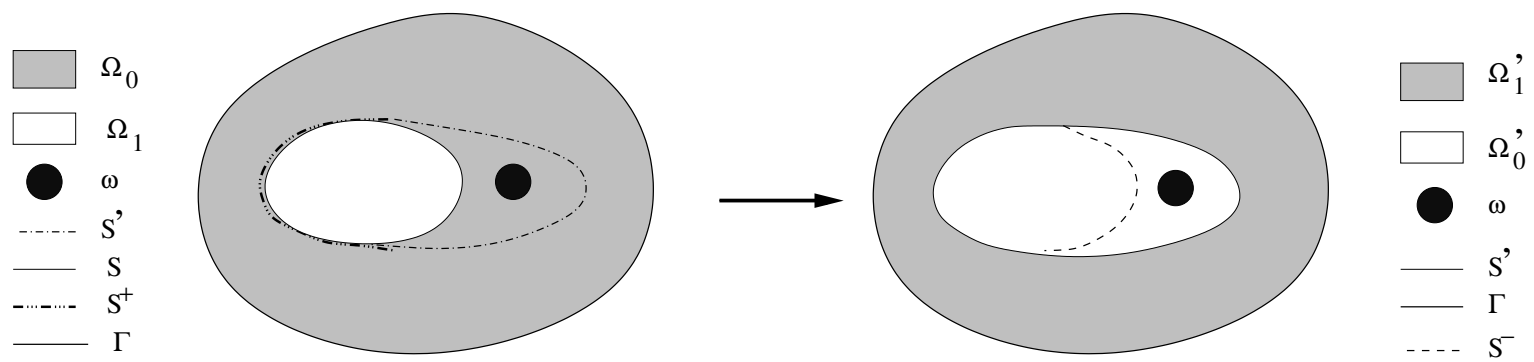

Figure 3. Case 2: Assumptions 2.2, 1.2 and 2.3 are fulfilled. The arrow means that we extend $S^{+}$to $S^{\prime}$.

\subsection{Carleman weight functions}

Carleman estimates on $q$ solution of (1.1) requires Carleman weight functions that we present now.

Lemma 2.1 Assume that we have the geometrical situation of case 1 . Under assumptions 1.2, 2.1, 2.3, 2.4, there exist functions $\beta_{i} \in \mathcal{C}^{0}(\bar{\Omega}), i=1,2$ that satisfy the following properties.

(i) $\beta_{i} \in \mathcal{C}^{0}(\bar{\Omega}), \beta_{i}^{k} \equiv \beta_{i \mid \Omega_{k}} \in \mathcal{C}^{2}\left(\overline{\Omega_{k}}\right), k=0,1 ; \operatorname{div}\left(a \nabla \beta_{i}\right) \in L^{\infty}(\Omega)$. (Thus the following transmission conditions are checked on $S:\left[\beta_{i}\right]=0$ and $\left.\left[a \partial_{n} \beta_{i}\right]=0\right)$.

(ii) $\beta_{i}(x)>0, \forall x \in \bar{\Omega}$.

(iii) There exists $r>0$ so that $\left|\nabla \beta_{i}\right| \geq r$ in $\Omega \backslash \omega, i=1,2$.

(iv) $\partial_{n} \beta_{i}(x)<0$ for any $x \in \Gamma, i=1,2$. 
Carleman estimates for the heat equation with discontinuous diffusion coefficients

(v) $\beta_{1}=\beta_{2}$ on $S^{00}, \beta_{1}(x)>\beta_{2}(x)$ for any $x \in S^{-}, \beta_{2}(x)>\beta_{1}(x)$ for any $x \in S^{+}$.

(vi) For $x \in S$, let the symmetrical matrix $\mathcal{M}(x):=\sum_{i=1,2} \mathcal{M}^{i}(x)$ of size $n+1$ where we set:

$$
\mathcal{M}^{i}:=\left(\begin{array}{ccc}
-\rho a \partial_{n} \beta_{i} & \nabla^{\prime} \beta_{i} & \left|\nabla^{\prime} \beta_{i}\right|^{2}-\rho\left(a \partial_{n} \beta_{i}\right)^{2} \\
{ }^{t} \nabla^{\prime} \beta_{i} & -\left(a \partial_{n} \beta_{i}\right) I_{n-1} & { }^{t} 0_{\mathbb{R}^{n-1}} \\
\left|\nabla^{\prime} \beta_{i}\right|^{2}-\rho\left(a \partial_{n} \beta_{i}\right)^{2} & 0_{\mathbb{R}^{n-1}} & 2 a \partial_{n} \beta_{i}\left\{\left|\nabla^{\prime} \beta_{i}\right|^{2}-\rho\left(a \partial_{n} \beta_{i}\right)^{2}\right\}
\end{array}\right) .
$$

Here $I_{n-1}$ denotes the unit matrix of size $n-1$ and $\rho(x):=\left(a_{0}(x) a_{1}(x)\right)^{-1}$.

Then $\mathcal{M}^{1} \leq 0$ on $S^{-}$and $\mathcal{M}^{2} \geq 0$ on $S^{+}$. Furthermore there exists a neighbourhood $V$ of $S^{00}$ in $S$ so that $\mathcal{M}=0$ on $V$.

Lemma 2.2 Assume that we have the geometrical situation of case 2. Under assumptions 1.2, 2.2, 2.3, 2.4, there exist functions $\beta_{i} \in \mathcal{C}^{0}(\bar{\Omega}), i=1,2$ that satisfy properties (i), (ii), (iv), (v), (vi) of lemma 2.1. The function $\beta_{2}$ satisfies (iii) of lemma 2.1 and $\beta_{1}$ satisfies:

(iii $)^{\prime}$ there exists $r>0$ so that $\left|\nabla \beta_{1}\right| \geq r$ in $\Omega \backslash(\omega \cup B)$, where $B$ is an opened ball such that $B \subset \subset \mathcal{O}$. Moreover $\beta_{1}$ and $\beta_{2}$ satisfy

$$
\text { (vii) } \beta_{2} \geq 2 \beta_{1} \text { in } B \text {. }
$$

Remark 2.4 In all cases 1, 2, we can construct $\beta_{i}$ so that $\beta_{i \mid \Gamma}$ are constant, $i=1,2$.

Remark 2.5 In all cases 1, 2, we shall construct $\beta_{i}, i=1,2$, so that in $V$ we have $\rho\left(a \partial_{n} \beta_{i}\right)^{2}-\left|\nabla^{\prime} \beta_{i}\right|^{2}=0, \mathcal{M}=0$, and

$$
\mathcal{M}^{i}=\left(\begin{array}{ccc}
-\rho a \partial_{n} \beta_{i} & \nabla^{\prime} \beta_{i} & 0 \\
{ }^{t} \nabla^{\prime} \beta_{i} & -\left(a \partial_{n} \beta_{i}\right) I_{n-1} & { }^{t} 0_{\mathbb{R}^{n-1}} \\
0 & 0_{\mathbb{R}^{n-1}} & 0
\end{array}\right)
$$

Thus the eigenvalues of $\mathcal{M}^{i}(x)$ are 0 (with multiplicity $n$ ) and $-(\rho(x)+n-1)\left(a \partial_{n} \beta_{i}\right)(x)$.

Lemmas 2.1 and 2.2 are proved in appendix.

\subsection{Global Carleman Estimates}

We set $\bar{\beta}:=\frac{5}{4} \max _{i=1,2} \max _{\bar{\Omega}} \beta_{i}$. We can assume that

$$
\max _{i=1,2} \max _{\bar{\Omega}} \beta_{i}(x)<\bar{\beta}<2 \min _{i=1,2} \min _{\bar{\Omega}} \beta_{i}(x),
$$

since if not, we replace $\beta_{i}$ by $\beta_{i}+4 \bar{\beta}$ and then $\bar{\beta}$ by $6 \bar{\beta}$.

We set

$$
\begin{aligned}
& \varphi_{i}(t, x):=\frac{\mathrm{e}^{\lambda \beta_{i}(x)}}{t(T-t)}, \eta_{i}(t, x):=\frac{\mathrm{e}^{\lambda \bar{\beta}}-\mathrm{e}^{\lambda \beta_{i}(x)}}{t(T-t)}, \\
& \Pi_{j}:=\sum_{i=1,2} s^{j} t^{-j}(T-t)^{-j} e^{-2 s \eta_{i}}, j \in \mathbb{N} .
\end{aligned}
$$

In order to state the main result of this work in common statements for cases 1 and 2 , we set $B=\mathcal{O}=\emptyset$ in case 1 . 
Theorem 2.1 Assume that conditions of lemma 2.1 in case 1 (respectively: of lemma 2.2 in case 2) are fulfilled. There exists $\lambda_{1}(\Omega, \omega, c)>0$ such that for each $\lambda>\lambda_{1}$ there exists a positive constant $C$ that only depend on $\Omega, \omega, \mathcal{O}$ and $a$, and $s_{1}(\lambda)>0$ so that the following estimate holds

$$
\int_{Q} \Pi_{3}|q|^{2}+\int_{Q} \Pi_{1}|\nabla q|^{2} \leq C\left(\int_{\omega_{T}} \Pi_{3}|q|^{2}+C \int_{Q} \Pi_{0}|L q|^{2}\right)
$$

for all $q \in D(L)$ and $s \geq s_{1}$. Moreover, $s_{1}$ is of the form $s_{1}=\sigma_{1}\left(T^{2}+T\right)$, where $\sigma_{1}$ is a positive constant that only depends on $\Omega, \omega, \mathcal{O}, a$ and $\lambda$.

Theorem 2.2 Assume that conditions of lemma 2.1 in case 1 (respectively: of lemma 2.2 in case 2) are fulfilled. Let $f \in\left(L^{2}(Q)\right)^{n}$ and $q \in L^{2}(Q)$ satisfying $\mathcal{L} q=$ divf, that is for any $g \in L^{2}(Q)$ we have

$$
\int_{Q} q g \mathrm{~d} x \mathrm{~d} t=-\int_{Q} f \cdot \nabla z \mathrm{~d} x \mathrm{~d} t+\int_{\Omega} q_{0} z\left(t_{0}\right) \mathrm{d} x,
$$

where $z \in D\left(L^{*}\right)$ is solution of $L^{*} z=g, z(T)=0$. Then there exists $\lambda_{1}(\Omega, \omega, a)>0$ such that for each $\lambda>\lambda_{1}$ there exists a positive constant $C$ that only depend on $\Omega, \omega$, and $a$, and $s_{1}(\lambda)>0$ so that the following estimate holds

$$
\int_{Q} \Pi_{3}|q|^{2} \leq C\left(\int_{\omega_{T}} \Pi_{3}|q|^{2}+C \int_{Q} \Pi_{2}|f|^{2}\right)
$$

for $s \geq s_{1}$. Moreover, $s_{1}$ is of the form $s_{1}=\sigma_{1}\left(T^{2}+T\right)$, where $\sigma_{1}$ is a positive constant that only depends on $\Omega, \omega$, ), $a$ and $\lambda$.

\subsection{Applications}

These Carleman estimates have many applications.

- Firstly, as described in [3], semilinear parabolic equations as

$$
\begin{cases}L q+f(q) & =v \chi_{\omega}+g \text { in } Q, \\ q(0, x) & =q_{0}(x) \text { in } \Omega,\end{cases}
$$

with unknown $q \in D(L)$ and under additional assumptions on the functional $f$ are exactly controllable to the trajectories. This is [3, theorem 2.1] if $S^{+}=\emptyset$.

- Secondly the inverse problem related to equation 1.1 and stated in terms of uniqueness and Hölder stability for $a(\cdot)$, as described in [4], is solved in our extended framework.

\subsection{Comments}

Global Carleman inequalities were first used in [5] to obtain inequality of observability for parabolic equations with $\mathcal{C}^{1}$ coefficients. By this approach the problem of null controllability for semilinear parabolic equations can be proved: see [6], [7], [8]. In the case of a discontinuous coefficient, results on global Carleman equalities and on null controllability recently appear thanks to the developments in [3]. In the $n$ dimensional 
case [9] gives a positive answer to the controllability question for a class of discontinuous coefficients, with separated variables that are smooth w.r.t. to all but one variables, which includes the case of stratified media. The proof relies on the global Carleman inequalities pointed in [9], [10] for the one - dimensional case. The proof for null controllability for the linear parabolic heat equation is then achieved in [11] for the case of a coefficient that exhibits jump of arbitrary sign at an interface. Our work is about Global Carleman inequalities for the heat equation as in [3] where the null controllability problem for semilinear parabolic equations is solved. In fact authors of [3] prove inequalities (2.8) and (2.10), but with some restrictions that we remove. Let us precise several points about this.

1. They assume that $a_{j} \in C^{2}\left(\overline{\Omega_{j}}\right), j=1,2$, which is too strong. Indeed, the Carleman functions they build do not depends on all the $a_{k}(x), x \in \Omega, k=0,1$, but only on the fraction $\left(a_{1} / a_{0}\right)(x), x \in S$. Their condition $-a_{j} \in C^{2}\left(\overline{\Omega_{j}}\right)$ - is only used in [3, Step 2, page 656] and similarly in [3, Step 4 page 659]. But if we use [12, Volume I, Corollary 1.3.4], then $a_{j} \in C^{1}\left(\overline{\Omega_{j}}\right), j=1,2$ suffices. Moreover, outside $S$, the assumption $a_{j} \in C^{1}\left(\overline{\Omega_{j}}\right)$ is only used to ensure that $\left(\operatorname{div}\left(a_{j} \nabla \beta\right)\right)_{\mid \Omega_{j}} \in C^{0}\left(\overline{\Omega_{j}}\right)$. But in fact that we only need is $\operatorname{div}(a \nabla \beta) \in L^{\infty}(\Omega)$. Hence the results (about Carleman estimates) in [3] still holds if the assumption $a_{j} \in C^{2}\left(\overline{\Omega_{j}}\right)$ is replaced by $a_{j} \in W^{1, \infty}\left(\Omega_{j}\right), j=0,1$.

2. They assume that $q$ belongs to the following restricted set $Z_{0} \subset D(L)$ :

$$
Z_{0}:=\left\{q: q_{\mid \Omega_{j}} \in \mathcal{C}^{2}\left(\left[t_{0}, T\right] \times \overline{\Omega_{j}}\right), j=0,1,[q]=0,\left[c \partial_{n} q\right]=0, q=0 \text { on } \Sigma\right\} .
$$

We can extend the results to $q \in D(L)$. See [4] for a proof.

3. They assume that $[a] \leq 0$, or equivalently $S^{+}=\emptyset$, in both cases 1,2 .

Besides, regarding the case 2 with $S^{+}=\emptyset$ and considering remark 2.1, assumption [3, Condition 2.2] appears more sophisticated than our assumption 2.2. (Precisely: two sets $-\mathcal{O}_{i}, i=1,2-$ in their work, only one $-\mathcal{O}-$ in our's) .

The paper is organized as follows. In Section 3 we first recall the main computations of [3] to prove (2.8) in the case 1, then we analyse the sign of the quadratic form coming from integrals on $S$. In Sections 4 and 5 we study two particular situations, where $S^{00}=\emptyset$. We show that we need only one function $\beta$ that we describe there.

In Section 6 we derive from section 3 and from [3] the proof for the case 2.

\section{Proof for the Case 1}

We prove the part of theorems 2.1, 2.2 corresponding to Case 1.

\subsection{Computation of integrals}

As in [3] we restrict the proof to $q \in Z_{0}$ defined above. The general case $q \in D(L)$ is completed by following the proof of $[4, \S 6.1]$. 
We particularly watch all the integrals on surfaces $\Gamma$ or $S$.

Let $q \in Z_{0}$ and $f:=L q \in L^{2}(Q)$. We set $\psi_{i}:=e^{-2 s \eta_{i}} q, i=1,2$. Then we have

$$
M_{1} \psi_{i}+M_{2} \psi_{i}=f_{i, s}
$$

where

$$
\begin{aligned}
& M_{1} \psi_{i}:=\operatorname{div}\left(a(x) \nabla \psi_{i}\right)+s^{2} \lambda^{2} \varphi_{i}^{2}\left|\nabla \beta_{i}\right|^{2} a(x) \psi_{i}+s \partial_{t} \eta_{i} \psi_{i}, \\
& M_{2} \psi_{i}:=\partial_{t} \psi_{i}-2 s \lambda \varphi_{i} a(x) \nabla \beta_{i} \cdot \nabla \psi_{i}-2 s \lambda^{2} \varphi_{i} a(x)\left|\nabla \beta_{i}\right|^{2} \psi_{i}, \\
& f_{i, s} \quad:=e^{-s \eta_{i}} f+s \lambda \varphi_{i} \operatorname{div}\left(a(x) \nabla \beta_{i}\right) \psi_{i}-2 s \lambda^{2} \varphi_{i} a(x)\left|\nabla \beta_{i}\right|^{2} \psi_{i} .
\end{aligned}
$$

From (3.1), we obtain

$$
\left\|M_{1} \psi_{i}\right\|_{2}^{2}+\left\|M_{2} \psi_{i}\right\|_{2}^{2}+2\left(M_{1} \psi_{i}, M_{2} \psi_{i}\right)=\left\|f_{i, s}\right\|_{2}^{2},
$$

where $(\cdot, \cdot)$ denotes the scalar product in $L^{2}(Q)$. We compute the scalar product in the left hand side of (3.2), with index $i$ omitted. We set $S_{T}=(0, T) \times S$ and $Q^{\prime}=Q \backslash S_{T}$. We have

$$
\left(M_{1} \psi, M_{2} \psi\right)=\sum_{k=1, l=1}^{3} I_{k l},
$$

where we set

- $I_{11}:=\int_{Q} \operatorname{div}(a(x) \nabla \psi) \partial_{t} \psi=0$,

- $I_{12}:=-2 s \lambda \int_{Q} \varphi \operatorname{div}(a(x) \nabla \psi) a(x) \nabla \beta \cdot \nabla \psi=J_{12}+K_{12}+H_{12}$,

where we set $J_{12}:=2 s \lambda \int_{Q^{\prime}} a \nabla(\varphi a \nabla \beta \cdot \nabla \psi) \cdot \nabla \psi, K_{12}:=2 s \lambda \int_{S_{T}} \varphi\left[\nabla \beta \cdot \nabla \psi a^{2} \partial_{n} \psi\right]$, $H_{12}:=-2 s \lambda \int_{\Sigma} \varphi \nabla \beta \cdot \nabla \psi a^{2} \partial_{n} \psi$. We compute

$$
\begin{aligned}
J_{12}= & 2 s \lambda^{2} \int_{Q^{\prime}} \nabla(\varphi a \nabla \beta \cdot \nabla \psi)(a \nabla \beta \cdot \nabla \psi)+2 s \lambda \int_{Q^{\prime}} \varphi a \partial_{j} \psi \partial_{j}\left(a \partial_{l} \beta\right) \partial_{l} \psi \\
& +2 s \lambda \int_{Q^{\prime}} \varphi a^{2} \partial_{j} \psi \partial_{j l}^{2} \psi \partial_{l} \beta \\
= & 2 s \lambda^{2} \int_{Q^{\prime}} \varphi|a \nabla \beta \cdot \nabla \psi|^{2}+2 s \lambda \int_{Q^{\prime}} \varphi a \partial_{j} \psi \partial_{j}\left(a \partial_{l} \beta\right) \partial_{l} \psi+s \lambda \int_{Q^{\prime}} \varphi a^{2} \nabla\left(|\nabla \psi|^{2}\right) \cdot \nabla \beta \\
= & 2 s \lambda^{2} \int_{Q^{\prime}} \varphi|a \nabla \beta \cdot \nabla \psi|^{2}+2 s \lambda \int_{Q^{\prime}} \varphi a \partial_{j} \psi \partial_{j}\left(a \partial_{l} \beta\right) \partial_{l} \psi \\
& -s \lambda \int_{Q^{\prime}}|\nabla \psi|^{2} \operatorname{div}\left(\varphi a^{2} \nabla \beta\right)+K_{12}^{\prime}+H_{12}^{\prime},
\end{aligned}
$$

where we set $K_{12}^{\prime}:=-s \lambda \int_{S_{T}} \varphi\left[|\nabla \psi|^{2} a^{2} \partial_{n} \beta\right], H_{12}^{\prime}:=s \lambda \int_{\Sigma}|\nabla \psi|^{2} \varphi a^{2} \partial_{n} \beta$. Finally since $H_{12}^{\prime}=-\frac{1}{2} H_{12}$ we get

$$
\begin{aligned}
& I_{12}:=-s \lambda^{2} \int_{Q} \varphi|a \nabla \beta|^{2}|\nabla \psi|^{2}+2 s \lambda^{2} \int_{Q} \varphi(a \nabla \psi \cdot \nabla \beta)^{2}+K_{12}+K_{12}^{\prime}+\frac{1}{2} H_{12}+X_{1}, \\
& X_{1}:=2 s \lambda \int_{Q^{\prime}} \varphi a \partial_{j} \psi \partial_{j}\left(a \partial_{l} \beta\right) \partial_{l} \psi-s \lambda \int_{Q^{\prime}} \varphi|\nabla \psi|^{2} \operatorname{div}\left(a^{2} \nabla \beta\right) .
\end{aligned}
$$$$
\text { - } I_{13}:=-2 s \lambda^{2} \int_{Q} \operatorname{div}(a \nabla \psi) \varphi a|\nabla \beta|^{2} \psi=2 s \lambda^{2} \int_{Q^{\prime}} a \nabla \psi \nabla\left(\varphi a|\nabla \beta|^{2} \psi\right)+K_{13} \text {, }
$$ 
with $K_{13}:=2 s \lambda^{2} \int_{S_{T}} \varphi\left[a^{2}|\nabla \beta|^{2} \psi \partial_{n} \psi\right]$. Thus we obtain

$$
I_{13}=2 s \lambda^{2} \int_{Q^{\prime}} \varphi|a \nabla \beta|^{2}|\nabla \beta|^{2}+X_{2}+K_{13}
$$

with $X_{2}:=2 s \lambda^{2} \int_{Q^{\prime}} \varphi a \nabla\left(a|\nabla \beta|^{2}\right) \nabla \psi \psi+2 s \lambda^{3} \int_{Q^{\prime}} \varphi a^{2}|\nabla \beta|^{2} \nabla \beta \cdot \nabla \psi \psi$.

- $I_{21}:=s^{2} \lambda^{2} \int_{Q} \varphi^{2}|\nabla \beta|^{2} a \partial_{t} \psi \psi=-s^{2} \lambda^{2} \int_{Q} \varphi \partial_{t} \varphi|\nabla \beta|^{2} a|\psi|^{2}$,

- $I_{22}:=-s^{3} \lambda^{3} \int_{Q} \varphi^{3} a^{2}|\nabla \beta|^{2} \nabla \beta \cdot \nabla \psi \psi=3 s^{3} \lambda^{4} \int_{Q} \varphi^{3} a^{2}|\nabla \beta|^{4}|\psi|^{2}+K_{22}+X_{3}$,

with $K_{22}:=s^{3} \lambda^{3} \int_{S_{T}} \varphi^{3}\left[a^{2}|\nabla \beta|^{2} \partial_{n} \beta\right]|\psi|^{2}, X_{3}:=s^{3} \lambda^{3} \int_{Q^{\prime}} \varphi^{3} \operatorname{div}\left(a^{2}|\nabla \beta|^{2} \nabla \beta\right)|\psi|^{2}$.

- $I_{23}:=-2 s^{3} \lambda^{4} \int_{Q} \varphi^{3} a^{2}|\nabla \beta|^{4}|\psi|^{2}$,

- $I_{31}:=s \int_{Q} \partial_{t} \eta \psi \partial_{t} \psi=-\frac{1}{2} \int_{Q} \partial_{t}^{2} \eta|\psi|^{2}$,

- $I_{32}:=-2 s^{2} \lambda \int_{Q} \varphi \partial_{t} \eta a \nabla \beta \cdot \nabla \psi \psi$

$$
=s^{2} \lambda \int_{Q^{\prime}} \varphi \operatorname{div}(a \nabla \beta)|\psi|^{2}+s^{2} \lambda^{2} \int_{Q^{\prime}} \varphi \partial_{t} \eta a|\nabla \beta|^{2}|\psi|^{2}-s^{2} \lambda^{2} \int_{Q^{\prime}} \varphi \partial_{t} \varphi a|\nabla \beta|^{2}|\psi|^{2}+K_{32}
$$

with $K_{32}:=s^{2} \lambda \int_{S_{T}} \varphi \partial_{t} \eta\left[a \partial_{n} \beta\right]|\psi|^{2}$.

- $I_{33}:=-2 s^{2} \lambda^{2} \int_{Q} \varphi a \partial_{t} \eta|\nabla \beta|^{2}|\psi|^{2}$.

\subsection{The quadratic form on the interface}

Thus we write (3.3) in the form $\left(M_{1} \psi_{i}, M_{2} \psi_{i}\right)=J_{i}+K_{i}+H_{i}$, with $J_{i}$ : integrals on $Q^{\prime}$, $K_{i}$ : integrals on $S_{T}, H_{i}$ : integrals on $\Sigma$. We have

$$
\begin{aligned}
J_{i}:= & \int_{S_{T}}\left\{Q_{n n}^{i}\left|a \partial_{n} \psi_{i}\right|^{2}+2 Q_{n \tau}^{i} \cdot \nabla^{\prime} \psi_{i}\left(a \partial_{n} \psi_{i}\right)+Q_{\tau \tau}^{i}\left|\nabla^{\prime} \psi_{i}\right|^{2}\right. \\
& \left.+\left(Q_{00}^{i}+X_{00}^{i}\right)\left|\psi_{i}\right|^{2}+2 X_{n 0}^{i}\left(a \partial_{n} \psi_{i}\right) \psi_{i}\right\}
\end{aligned}
$$

with

$Q_{n n}^{i}:=s \lambda \varphi_{i}\left[\partial_{n} \beta_{i}\right], \quad Q_{n \tau}^{i}:=s \lambda \varphi_{i}\left[a \nabla^{\prime} \beta_{i}\right], \quad Q_{\tau \tau}^{i}:=-s \lambda \varphi_{i}\left[a^{2} \partial_{n} \beta_{i}\right]$,

$X_{n 0}^{i}:=s \lambda^{2} \varphi_{i}\left[a\left|\nabla \beta_{i}\right|^{2}\right], Q_{00}^{i}:=s^{3} \lambda^{3} \varphi_{i}^{3}\left[a^{2} \partial_{n} \beta_{i}\left|\nabla \beta_{i}\right|^{2}\right], X_{00}^{i}:=s^{2} \lambda \varphi_{i} \partial_{t} \eta_{i}\left[a \partial_{n} \beta_{i}\right]$.

We write (3.4) by using $\psi_{i}:=e^{-s \eta_{i}} q$ and $\nabla \psi_{i}=e^{-s \eta_{i}} \nabla q+s \lambda \varphi_{i} \nabla \beta_{i} q$. Thus we have

$$
\begin{aligned}
J_{i}:= & \int_{S_{T}} e^{-2 s \eta_{i}}\left\{Q_{n n}^{i}\left|a \partial_{n} q\right|^{2}+2 Q_{n \tau}^{i} \cdot \nabla^{\prime} q\left(a \partial_{n} q\right)+Q_{\tau \tau}^{i}\left|\nabla^{\prime} q\right|^{2}\right. \\
& \left.+\left(2 Q_{00}^{i}+Y_{00}^{i}\right)|q|^{2}+2\left(Q_{n 0}^{i}+X_{n 0}^{i}\right)\left(a \partial_{n} q\right) q\right\},
\end{aligned}
$$

with $Y_{00}^{i}:=2 s^{-1} \varphi_{i}^{-1} Q_{00}^{i}+X_{00}^{i}, Q_{n 0}^{i}:=s^{2} \lambda^{2} \varphi_{i}^{2}\left[a\left|\nabla \beta_{i}\right|^{2}\right]$. We set

$$
R^{i}:=\left(\begin{array}{ccc}
Q_{n n}^{i} & Q_{n \tau}^{i} & Q_{n 0}^{i} \\
Q_{n \tau}^{i} & Q_{\tau \tau}^{i} I_{n-1} & { }^{t} 0_{\mathbb{R}^{n-1}} \\
Q_{n 0}^{i} & 0_{\mathbb{R}^{n-1}} & 2 Q_{00}^{i}
\end{array}\right), X^{i}:=\left(\begin{array}{ccc}
0 & 0_{\mathbb{R}^{n-1}} & X_{n 0}^{i} \\
{ }^{t} 0_{\mathbb{R}^{n-1}} & 0_{n-1} & { }^{t} 0_{\mathbb{R}^{n-1}} \\
X_{n 0}^{i} & 0_{\mathbb{R}^{n-1}} & Y_{00}^{i}
\end{array}\right),
$$


and $R_{i}^{\prime}:=e^{-2 s \eta_{i}} R^{i}, X_{i}^{\prime}:=e^{-2 s \eta_{i}} X^{i}, R^{\prime}:=\sum_{i=1,2} R_{i}^{\prime}, X^{\prime}:=\sum_{i=1,2} X_{i}^{\prime}, P:=R^{\prime}+X^{\prime}$. We prove that $P \geq 0$ on $S$ for $s$ sufficiently large.

Firstly, note that if $x \in S^{0}$ then $P=0_{n+1}$, since $[a(x)]=0$.

Let $L^{i}(x), i=1,2$, be the $(n+1)$ sized strictly positive diagonal matrix with $L_{j j}^{i}=1$, $j \leq n$, and $L_{n+1, n+1}^{i}(x)=s \lambda \varphi_{i}(x)$ for $x \in S$. Thus $R^{i}=[a] s \lambda \varphi_{i} L^{i} \mathcal{M}^{i} L^{i}$.

Let $x \in V \cap S^{+}$. Note that $X_{00}^{i}=0$ since $\left[a \partial_{n} \beta_{i}(x)\right]=0$, and that $X_{n 0}^{i}=Q_{00}^{i}=Y_{00}^{i}=0$. Thus $X^{\prime}(x)=0_{n+1}$. Since $[a(x)] \geq 0, \mathcal{M}^{2}(x) \geq 0$ and $\mathcal{M}^{1}(x)=-\mathcal{M}^{2}(x) \leq 0$, we thus have $R^{2}(x) \geq 0$ and $R^{1}(x) \leq 0$. From $\beta_{2}(x) \geq \beta_{1}(x)$ we obtain $\varphi_{2}(x) \geq \varphi_{1}(x)$, $\eta_{2}(x) \leq \eta_{1}(x), e^{-2 s \eta_{2}(x)} \geq e^{-2 s \eta_{1}(x)}$ and $L^{2}(x) \geq L^{1}(x)$. We thus obtain $R_{2}^{\prime}(x) \geq-R_{1}^{\prime}(x)$, $R^{\prime}(x) \geq 0$, and $P(x) \geq 0$.

Similarly we obtain $P(x) \geq 0$ for any $x \in V \cap S^{-}$.

Let $x \in S^{+} \backslash V$. We have $\mathcal{M}^{2}(x) \geq r>0$ for some $r \geq 0$ that do not depend of $x$ and $X^{2}=[a] \lambda L^{2} \mathcal{Y}^{2} L^{2}$ where the matrix $\mathcal{Y}^{2}$ does not depend on $s$ and $\lambda$. So $s^{-1}\left|\mathcal{Y}^{2}(x)\right| \leq \frac{1}{4} \mathcal{M}^{2}(x)$ for $s$ sufficiently large, $X^{2}$ is a perturbation term for $R^{2}$ of order $s^{-1} \varphi_{2}^{-1}$, and $R_{2}^{\prime}+X_{2}^{\prime} \geq \frac{3}{4} R_{2}^{\prime}$. Then we have $\mathcal{M}^{2}(x) \geq r>0$ and $\beta_{2}(x) \geq \beta_{1}(x)+r$ for some $r \geq 0$ that does not depend of $x$. Thus $L^{2}(x) \geq L^{1}(x)$ and $e^{-2 s \eta_{1}(x)}$ is a perturbation term for $e^{-2 s \eta_{2}(x)}$ of order $s^{-1}$ (at least). Consequently we have $R_{2}^{\prime}(x)+R_{1}^{\prime}(x)+X_{2}^{\prime}(x) \geq \frac{1}{4} R_{2}^{\prime}(x)$ if $s$ is sufficiently large, uniformly for $x \in S^{+} \backslash V$. Thus $P(x) \geq 0$.

Similarly we obtain $P(x) \geq 0$ for any $x \in S^{-} \backslash V$.

Consequently, if $s$ is sufficiently large, then $\sum_{i=1,2} J_{i} \geq 0$.

Then we can complete the computations in [3] and the proof of (2.8).

\section{A Particular situation for the case 1}

The following result corresponds to the case $S^{-}=\emptyset$.

Lemma 4.1 We assume that $\Gamma$ and $S$ are of class $\mathcal{C}^{2}$ and $S^{+}=S \backslash S^{0}$ satisfies assumption 2.3. Then there exists a function $\beta \in \mathcal{C}^{0}(\bar{\Omega})$ that satisfies the following properties.

(1) $\beta^{k} \equiv \beta_{\mid \Omega_{k}} \in \mathcal{C}^{2}\left(\overline{\Omega_{k}}\right), k=0,1$;

(2) $[\beta]=0$ and $\left[a \partial_{n} \beta_{i}\right]=0$;

(3) $\beta(x)>0, \forall x \in \bar{\Omega}$.

(4) There exists $r>0$ so that $|\nabla \beta| \geq r$ in $\Omega \backslash \omega$.

(5) $\beta$ is constant on $\Gamma$ and $\partial_{n} \beta(x)<0$ for any $x \in \Gamma$.

(6) There exists $r>0$ so that if we set

$$
\mathcal{M}(x):=\left(\begin{array}{ccc}
-\rho a \partial_{n} \beta & \nabla^{\prime} \beta_{i} & \left|\nabla^{\prime} \beta\right|^{2}-\rho\left(a \partial_{n} \beta\right)^{2} \\
{ }^{t} \nabla^{\prime} \beta & -\left(a \partial_{n} \beta\right) I_{n-1} & { }^{t} 0_{\mathbb{R}^{n-1}} \\
\left|\nabla^{\prime} \beta\right|^{2}-\rho\left(a \partial_{n} \beta\right)^{2} & 0_{\mathbb{R}^{n-1}} & 2 a \partial_{n} \beta\left\{\left|\nabla^{\prime} \beta\right|^{2}-\rho\left(a \partial_{n} \beta\right)^{2}\right\}
\end{array}\right),
$$

then $\mathcal{M}(x) \geq r>0$ on $S^{+}$. 
We prove this lemma in appendix.

Remark 4.1 We built $\beta$ independently of the fact that $[a]=0$ in $S \backslash S^{+}$. This will be used above (see the Proof of lemma 2.1).

We straightforwardly obtain:

Corollary 4.1 If we modify the weight functions by setting

$$
\begin{aligned}
& \varphi(t, x):=\frac{\mathrm{e}^{\lambda \beta(x)}}{t(T-t)}, \eta(t, x):=\frac{\mathrm{e}^{\lambda \bar{\beta}}-\mathrm{e}^{\lambda \beta(x)}}{t(T-t)}, \\
& \Pi_{j}:=s^{j} t^{-j}(T-t)^{-j} e^{-2 s \eta}, j \in \mathbb{N},
\end{aligned}
$$

then theorems 2.1 and 2.2 still hold in case 1 with $S^{-}=\emptyset$.

\section{A second Particular situation for the case 1}

In this part we assume $S^{-}$and $S^{+}$non empty, and $S^{00}$ empty, that is, $\mathrm{d}\left(S^{-}, S^{+}\right)=0$. Thus for any $\varepsilon \in(0,1)$ there exists two open sets $U_{1}, U_{2} \subset \subset \Omega$ with $\mathrm{d}\left(U_{1}, U_{2}\right)>0$, $S^{+} \subset U_{2}, S^{-} \subset U_{1}$, and two functions $\psi_{1}, \psi_{2} \in \mathcal{C}^{2}(\bar{\Omega})$ such that $\varepsilon \leq \psi_{i} \leq 1, \psi_{1}=\varepsilon$ in $S^{+}, \psi_{1}=1$ in $\Omega \backslash U^{2}, \psi_{2}=\varepsilon$ in $S^{-}, \psi_{2}=1$ in $\Omega \backslash U^{2}$,

Let $\beta_{2}=\psi_{2} \beta$ where $\beta$ is built as in lemma 4.1 (with the modification $S^{+}=S \backslash\left(S^{-} \cup S^{0}\right)$ ). See also remark 4.1. Let $\beta_{1}=\psi_{1} \beta$ where $\beta$ is defined as in [3, (43) page 629] (with the modification that $S^{+}$is non empty). See also remark 4.1 .

We choose $\varepsilon>0$ such that $\beta_{1} \geq \frac{1}{2} \beta_{2}$ in $U_{1}$ and $\beta_{2} \geq \frac{1}{2} \beta_{1}$ in $U_{2}$. Note that $\left|\nabla \beta_{1}\right| \geq r>0$ in $\Omega \backslash\left(\omega \cup U_{2}\right)$ and $\left|\nabla \beta_{2}\right| \geq r>0$ in $\Omega \backslash\left(\omega \cup U_{1}\right)$. Thus $\left|\nabla \beta_{1}\right|+\left|\nabla \beta_{2}\right| \geq r>0$ in $\Omega \backslash \omega$. Then we can easily check that

$$
J:=\sum_{i=1,2} J_{i} \geq r \int_{S_{T}} s \lambda \varphi_{i} e^{-2 s \eta_{i}}|[a]|\left\{\left|a \partial_{n} q\right|^{2}+\left|\nabla^{\prime} q\right|^{2}+s^{2} \lambda^{2} \varphi^{2}|q|^{2}\right\} .
$$

for some $r>0$ and for $s$ sufficiently large. Hence with this modified function $\beta$, the conclusion of corollary 4.1 holds again in this situation.

\section{Proof for the case 2}

We prove the part of theorems 2.1, 2.2 corresponding to Case 2.

Thanks to the computations in [3, Proof of theorem 3.4] and to the properties of the Carleman weights that originate from lemma 2.2, we can complete the proof of these theorems in the case 2 .

Remark 6.1 If $S^{-}$is empty or, more generally, if $S^{00}$ is empty, then we are as in the first or the second particular situations above - see sections 4, 5 but corresponding to case 2. Then, by following lemma 4.1 and section 5, we can prove theorems 2.1 and 2.2 (in case 2) by introducing only one function $\beta$. This is as corollary 4.1, but in the situation of case 2. 


\section{Appendix}

\subsection{Proof of lemma 4.1}

Step 1 - Let $S^{\prime}$ be an extension of $S^{+}$inside $\Omega_{0}$ of class $\mathcal{C}^{2}$, that separates $\Omega$ in two open sets $\Omega_{0}^{\prime}$ and $\Omega_{1}^{\prime}$, with $\omega \subset \subset \Omega_{0}^{\prime} \subset \subset \Omega$. We denote by $n^{\prime}$ the unit vector field on $S^{\prime}$ with $n^{\prime}(x)$ pointing towards $\Omega_{0}^{\prime}$. We are thus in the situation of [3, case 2, page 1] with the prime suppressed, but $a_{j} \in W^{1, \infty}\left(\Omega_{j}\right)$ only (that is not a problem as we pointed in comment 1. of $\S 2.5)$. So at this point we could conclude with two $\beta_{j}$ functions. In fact, we can use only one. This has a the great advantadge for the inverse problem treatment: one measurement instead of two (see [4]).

Indeed, we construct a function $\beta^{\prime} \in \mathcal{C}^{0}\left(\overline{\Omega_{0}^{\prime}}\right)$ satisfying

- $\beta^{\prime}(x)>0, \forall x \in \overline{\Omega_{0}^{\prime}}$,

- $\beta_{\mid \Gamma}^{\prime}$ is constant and $\partial_{n} \beta^{\prime}(x)<0$ for any $x \in \Gamma$,

- $\beta_{\mid S^{\prime}}^{\prime}$ is constant and $\partial_{n^{\prime}} \beta^{\prime}(x)>0$ for any $x \in S^{\prime}$,

- There exists $r>0$ such that $\left|\nabla \beta^{\prime}\right| \geq r$ in $\Omega_{0}^{\prime} \backslash \omega$.

All these points can be checked by following [3, Proof of lemma 3.2, Steps 4, 5, 6]. (Concerning the last point, we use there the fact that each connected component of $\Omega_{0}^{\prime}$ contains part of $\omega$, thanks to assumption 2.3).

Note that we have $\partial_{n} \beta^{\prime}(x)<0$ for any $x \in S \cap S^{\prime}$, since $n^{\prime}=-n$ on $S \cap S^{\prime}$.

We set $\beta=\beta^{\prime}$ in $\overline{\Omega_{1}}$.

Step 2 - Following [3, Proof of Lemma 3.1, Steps 2, 3, 4], we then extend $\beta$ in $\Omega_{0}$ such that

(i) $\beta \in \mathcal{C}^{0}(\bar{\Omega}) ; \beta^{k} \equiv \beta_{\mid \Omega_{k}} \in \mathcal{C}^{2}\left(\overline{\Omega_{k}}\right), k=0,1 ; \partial_{n} \beta^{0}=\frac{a_{1}}{a_{0}} \partial_{n} \beta^{1}$ on $S$. (Thus $\beta$ satisfies the transmission conditions on $S:[\beta]=0$ and $\left.\left[a \partial_{n} \beta\right]=0\right)$.

(ii) $\beta(x)>0, \forall x \in \bar{\Omega}$.

(iii) There exists $r>0$ so that $|\nabla \beta| \geq r$ in $\Omega \backslash \omega$.

(Concerning point (iii), we use there the fact that each connected component of $\Omega_{0}$ contains part of $\omega$, thanks to assumption 1.2).

We thus have $\nabla^{\prime} \beta(x)=0$ for any $x \in \Gamma \cup S^{+}$, and, for any $x \in S^{+} \subset S \cap S^{\prime}$, $\mathcal{M}(x) \geq r^{\prime}>0$. (Here, setting $\alpha \equiv a \partial_{n} \beta$, the value

$$
r^{\prime}=\min _{x \in \overline{S^{+}}} \alpha(x) \min \left(1, \rho(x) \frac{\alpha^{2}(x)}{1+2 \alpha^{2}(x)}\right)
$$

is suitable).

\subsection{Proof of lemma 2.1}

We will use the following lemma: 
Lemma 7.1 Let $r>0$ and, for any $\varepsilon$ sufficiently small, let $\phi_{\varepsilon}$ be a $\mathcal{C}^{2}$ diffeomorphism defined in a neighbourhood $\bar{U}$ of $S$ such that $\left\|\phi_{\varepsilon}-I d\right\|_{\infty}<\varepsilon,\left|J\left(\phi_{\varepsilon}\right)-1\right|<\varepsilon$, where $J\left(\phi_{\varepsilon}\right)$ denotes the jacobian of $\phi_{\varepsilon}$. We assume that $S_{\varepsilon}:=\phi_{\varepsilon}(S) \subset U_{1}, U_{1}:=U \cap \Omega_{1}$, and we denote by $n_{\varepsilon}$ the unit normal on $S_{\varepsilon}$ pointing towards $S$.

Let $h_{1}, h_{2}$ be two functions in $\mathcal{C}^{2}\left(\overline{\Omega_{1}}\right)$ with $h_{1}-h_{2} \circ \phi_{\varepsilon} \geq r, g_{1} \geq r$ on $S$ and $g_{2} \geq r$ on $S_{\varepsilon}$, where we set $g_{1}:=\partial_{n} h_{1}$ on $S, g_{2}=\partial_{n_{\varepsilon}} h_{2}$ on $S_{\varepsilon}$.

Then there exists $\varepsilon_{0}>0$ such that for any $\varepsilon \in\left(0, \varepsilon_{0}\right]$ there exist in $\overline{U_{1}}$ a neighbourhood $\overline{U_{\varepsilon}}$ of $S$ containing $S_{\varepsilon}$ and a function $h \in C^{2}\left(\overline{U_{\varepsilon}}\right)$ so that $h=h_{1}$ on $S, h=h_{2}$ on $S_{\varepsilon}$, $\partial_{n} h=g_{1}$ on $S, \partial_{n_{\varepsilon}} h=g_{2}$ on $S_{\varepsilon}$, and $|\nabla h| \geq \frac{1}{2} r$ for any $x \in \overline{U_{\varepsilon}}$.

\section{Proof}

Denote by $p$ the orthogonal projection on $S$, defined in a neighbourhood $U^{\prime}$ of $S$ in $\Omega$. Then $p \in \mathcal{C}^{2}\left(U^{\prime}\right)$. We set $q:=I d-p \in \mathcal{C}^{2}\left(U^{\prime}\right)$. Let $U_{\varepsilon}:=\{t p(x)+(1-t) x$; $\left.0<t<1 ; x \in S_{\varepsilon}\right\}$. Note that for any $z \in \overline{U_{\varepsilon}}$ there exists an unique $(t, x) \in[0,1] \times S_{\varepsilon}$ so that $z=t p(x)+(1-t) x$, and $z \mapsto(t, x)$ is of class $C^{2}$. We set $\psi \in \mathcal{C}_{0}^{\infty}([-1,1])$ such that $0 \leq \psi \leq 1, \psi=1$ in $\left[0, \frac{1}{4}\right], \psi=0$ in $\left[\frac{3}{4}, 1\right]$. We set $h(z)=(1-\psi(t)) h_{1}(z)+\psi(t) h_{2}(z)$. Thus $h=h_{1}$ (respectively $h=h_{2}$ ) on a neighbourhood of $S$ (respectively $S_{\varepsilon}$ ). Moreover we have $\partial_{t} h(z)=-\left((1-\psi(t)) \nabla h_{1}(z)+\psi(t) \nabla h_{2}(z)\right) \cdot q(x)+\psi^{\prime}(t)\left(h_{2}(z)-h_{1}(z)\right)$. Since $h_{1}-h_{2} \circ \phi_{\varepsilon} \geq r>0$ on $S$, then there exists $\varepsilon_{0}>0$ so that $h_{1}(z)>h_{2}\left(z^{\prime}\right)$ for all $\varepsilon \in\left(0, \varepsilon_{0}\right)$ and all $z, z^{\prime} \in \overline{U_{\varepsilon}}$. On the other hand if $x \in S_{\varepsilon}$ then $q(x)=-|q(x)| n(p(x))$, $|q(x)|=O(\varepsilon)$ as $\varepsilon \rightarrow 0$ and $z=t p(x)+(1-t) x=p(x)+(1-t) q(x)=p(x)+O(\varepsilon)$. Thus we have $\nabla h_{1}(z) \cdot q(x)=-g_{1}(p(x))|q(x)|(1+O(\varepsilon))<0$ and $\nabla h_{2}(z) \cdot q(x)=$ $-g_{2}(x)|q(x)|(1+O(\varepsilon))<0$ as $\varepsilon \rightarrow 0$. Hence if $\varepsilon$ is sufficiently small we obtain $\partial_{t} h(z) \geq \frac{1}{2} r$ for any $z \in \overline{U_{\varepsilon}}$.

Let us prove lemma 2.1 in 6 steps. We successively construct $\beta_{2 \mid S^{\prime}},\left(a \partial_{n^{\prime}} \beta_{2}\right)_{\mid S^{\prime}}, \beta_{2}$ in $\Omega$, $\beta_{1 \mid S},\left(a \partial_{n} \beta_{1}\right)_{\mid S}, \beta_{1}$ in $\Omega$.

Step 1 - Let $S^{\prime}$ be the interface between $\Omega_{0}^{\prime}$ and $\Omega_{1}^{\prime}$ that extends $S^{+}$inside $\Omega_{0}$. Thus $S^{00} \subset S \cap S^{\prime}$. Let $h_{0}$ be the function of assumption 2.4 defined in $V \subset S$ and let $\tilde{h}_{0} \in \mathcal{C}^{2}(W)$ be an extension to $W \subset \Omega$, as explained in remark 2.3.

We set $V^{\prime}=W \cap S^{\prime}$ of $S^{00}$ and $h^{\prime}=2+\tilde{h}_{0}$ in $V^{\prime}$. Then $h^{\prime}$ can be extended to $S^{\prime}$ so that

- $h^{\prime} \in \mathcal{C}^{2}\left(S^{\prime}\right)$;

- $3 \geq h^{\prime}(x)>0$ for all $x \in S^{\prime}$;

- $h^{\prime}(x)>2$ for all $x \in S^{+}$.

Note that $h^{\prime}=2$ on $S^{00}, h^{\prime}(x)<2$ for all $x \in V \cap S^{-}$, and $\nabla^{\prime} h^{\prime}(x) \neq 0$ for all $x \in V^{\prime}$. We then consider $g^{\prime} \in \mathcal{C}^{1}\left(S^{\prime}\right)$ defined on $V^{\prime}$ by

$$
g^{\prime}=\rho^{-\frac{1}{2}}\left|\nabla^{\prime} h^{\prime}\right|,
$$

and satisfying $g^{\prime}(x)>0$ for all $x \in S^{\prime}$. 
Step 2 - Corollary 1.3.4 of [12, Volume I] shows that there exists a positive function $\beta^{\prime}$ defined in a neighbourhood $U_{0} \subset \overline{\Omega_{0}^{\prime}}$ of $S^{\prime}$ with $\beta^{\prime} \in C^{2}\left(U_{0}\right), \beta_{\mid S^{\prime}}^{\prime}=h^{\prime}$ and $\partial_{n^{\prime}} \beta^{\prime}=\frac{1}{a_{1}} g^{\prime}$ on $S^{\prime}$.

Thanks to assumption 2.3 and as in Proof of Lemma 4.1, Step 1, we then extend $\beta^{\prime}$ into $\Omega_{0}^{\prime}$, so that

- $\beta^{\prime} \in \mathcal{C}^{0}\left(\overline{\Omega_{0}^{\prime}}\right)$;

- $\beta^{\prime}(x)>0$ for any $x \in \overline{\Omega_{0}^{\prime}}$;

- $\beta_{\mid \Gamma}^{\prime}$ is constant and $\partial_{n} \beta^{\prime}(x)<0$ for any $x \in \Gamma$.

- There exists $r>0$ so that $\left|\nabla \beta^{\prime}\right| \geq r$ in $\Omega_{0}^{\prime} \backslash \omega$.

We set $\beta_{2}=\beta^{\prime}$ in $\overline{\Omega_{1}}$.

Step 3 - Thanks to assumption 1.2 and as in Proof of Lemma 4.1, Step 2, we then extend $\beta_{2}$ into $\Omega_{0}$, so that

- $\beta_{2} \in \mathcal{C}^{0}(\bar{\Omega}), \beta_{2 \mid \Omega_{k}} \in \mathcal{C}^{2}\left(\overline{\Omega_{k}}\right)$;

- $\beta_{2}(x)>0$ for any $x \in \bar{\Omega}$;

- $\beta_{2 \mid \Gamma}$ is constant and $\partial_{n} \beta_{2}(x)<0$ for any $x \in \Gamma$.

- There exists $r>0$ so that $\left|\nabla \beta_{2}\right| \geq r$ in $\Omega \backslash \omega$.

- $\left[a \partial_{n} \beta_{2}\right]=0$ on $S$.

Note that $\left(a \partial_{n} \beta_{2}\right)(x)=-g^{\prime}(x)<0$ for any $x \in \overline{S^{+}}$.

Step 4 - Thanks to the properties of $h^{\prime}$ (cf. step 1) we have $3 \geq \beta_{2}(x)>2$ for $x \in S^{+}$ and $\beta_{2}(x)<2$ for $x \in V \cap S^{-}$. Thus there exists $h \in \mathcal{C}^{2}(S)$ with the following properties:

- $h=4-\beta_{2}$ in $\overline{S^{+}} \cup V$;

- $h(x)>\beta_{2}(x)$ for all $x \in S^{-}$,

- $h(x)>0$ for all $x \in S$.

Note that $h=2$ on $S^{00}, h(x)<\beta_{2}(x)$ for any $x \in S^{+}, h(x)>\beta_{2}(x)$ for any $x \in S^{-}$, and $\nabla^{\prime} \beta_{2}(x) \neq 0$ for all $x \in V$. We then consider $g \in \mathcal{C}^{1}(S)$ defined in $V$ by

$$
g=\rho^{-\frac{1}{2}}\left|\nabla^{\prime} h\right|
$$

and satisfying $g(x)>0$ for all $x \in S$. Note that $g=\rho^{-\frac{1}{2}}\left|\nabla^{\prime} \beta_{2}\right|$ in $V$.

Step 5 - Corollary 1.3.4 of [12, Volume I] implies that there exists a positive function $\beta$ defined in a neighbourhood $U \subset \overline{\Omega_{0}}$ of $S$ with $\beta \in C^{2}(U), \beta_{\mid S}=h$ and $\partial_{n} \beta=\frac{1}{a_{0}} g$ on $S$.

Thanks to assumption 1.2 and as in Proof of Lemma 4.1, Step 2, we then extend $\beta$ into $\Omega_{0}$ so that

- $\beta \in \mathcal{C}^{2}\left(\overline{\Omega_{0}}\right)$;

- $\beta(x)>0$ for any $x \in \overline{\Omega_{0}}$;

- There exists $r>0$ so that $|\nabla \beta| \geq r$ in $\Omega_{0} \backslash \omega$. 
Step 6 - Corollary 1.3.4 of [12, Volume I] implies that there exists a positive function $\alpha$ defined in a neighbourhood $\overline{U_{1}} \subset \overline{\Omega_{1}}$ of $S$ with $\alpha \in C^{2}\left(\overline{U_{1}}\right), \alpha_{\mid S}=h$ and $\partial_{n} \alpha=g$ on $S$. Moreover we can choose $U_{1}$ so that $\alpha(x)>0$ and $\nabla \alpha(x) \neq 0$ for $x \in \overline{U_{1}}$.

For $\varepsilon>0$ we set $V_{\varepsilon}^{1}:=\left\{x \in \overline{\Omega_{1}} ; d(x, S)<\varepsilon\right\}, \Omega_{1}^{\varepsilon}=\Omega_{1} \backslash \overline{V_{\varepsilon}^{1}}$ and $S_{\varepsilon}:=\left\{x \in \overline{\Omega_{1}}\right.$; $d(x, S)=\varepsilon\}$. We notice that if $\varepsilon$ is sufficiently small then $S_{\varepsilon}$ satisfies the condition in lemma 7.1 (with some $\phi_{\varepsilon}$ ) and $\left(\Omega_{1}^{\varepsilon}, S_{\varepsilon}\right)$ satisfies assumption 2.1. The proof of [3, Lemma 3.1, Step 1] with $\left(\Omega_{1}, S\right)$ replaced by $\left(\Omega_{1}^{\varepsilon}, S_{\varepsilon}\right)$ shows that there exists a function $\beta_{3} \in \mathcal{C}^{2}\left(\overline{\Omega_{1}^{\varepsilon}}\right)$ such that

- $\beta_{3}(x)>0$ for any $x \in \overline{\Omega_{1}^{\varepsilon}}$;

- $\beta_{3 \mid \Gamma}$ is constant and $\partial_{n} \beta_{3}(x)<0$ for any $x \in \Gamma$;

- $\beta_{3 \mid S_{\varepsilon}}=\frac{1}{2} \min _{\overline{U_{1}}} \alpha$ on $S_{\varepsilon}$.

- There exists $r^{\prime}>0$ so that $\left|\nabla \beta_{3}\right| \geq r^{\prime}$ in $\overline{\Omega_{1}^{\varepsilon}}$.

Moreover, we can extend $\beta_{3}$ in $V_{\varepsilon}^{1}$ in such a way that $\beta_{3} \in \mathcal{C}^{2}\left(\overline{\Omega_{1}}\right)$ and $\beta_{3}\left(\phi_{\varepsilon}(x)\right)<\alpha(x)$ for $x \in S$. Then we apply lemma 7.1 with $h_{1}=\alpha, h_{2}=\beta_{3}$, and we denote by $h_{3} \in \mathcal{C}^{2}\left(\overline{U_{\varepsilon}}\right)$ the resulting function $h$ of lemma 7.1.

We set $\beta_{1}=\beta_{3}$ in $\overline{\Omega_{1}^{\varepsilon}}$ and $\beta_{1}=h_{3}$ in $V_{\varepsilon}^{1} \subset \overline{U_{\varepsilon}}$. In fact, in such a way, the property $\beta_{1 \mid \Omega_{1}} \in \mathcal{C}^{2}\left(\Omega_{1}\right)$ fails, but we can use the argument at the end of [3, proof of Lemma 3.1, Step 1]. This shows that we can extend $\beta_{1 \mid \Omega_{0}}$ into $\Omega_{1}$ as we wish.

\subsection{Proof of lemma 2.2}

We follow the proof of lemma 2.1 by pointing the modifications. We consider an interface $S^{\prime}$ between $\Omega_{0}^{\prime}$ and $\Omega_{1}^{\prime}$ that extends $S^{+}$inside $\Omega_{0}$.

Step 1 - We construct a neighbourhood $V^{\prime} \subset S^{\prime}$ of $S^{00}, h^{\prime} \in \mathcal{C}^{2}\left(S^{\prime}\right)$ and $g^{\prime} \in \mathcal{C}^{1}\left(S^{\prime}\right)$ so that

- $h^{\prime}=2$ on $S^{00}$,

- $3 \geq h^{\prime}(x)>0$ for all $x \in S^{\prime}$,

- $h^{\prime}(x)>2$ for all $x \in S^{+}$,

- $h^{\prime}(x)<2$ for all $x \in V \cap S^{-}$,

- $g^{\prime}=\rho^{-\frac{1}{2}}\left|\nabla^{\prime} h^{\prime}\right|$ in $V^{\prime}$,

- $g^{\prime}(x)>0$ for any $x \in S^{\prime}$.

Step 2 - Thanks to assumption 2.3, there exists a positive function $\beta^{\prime}$ defined in $\Omega_{0}^{\prime}$ so that

- $\beta^{\prime} \in \mathcal{C}^{0}\left(\overline{\Omega_{0}^{\prime}}\right)$ and $\beta^{\prime}(x)>0$ for all $x \in \overline{\Omega_{0}^{\prime}}$;

- There exists $r>0$ so that $\left|\nabla \beta^{\prime}\right| \geq r$ in $\Omega_{0}^{\prime} \backslash \omega$.

We set $\beta_{2}=\beta^{\prime}$ in $\overline{\Omega_{1}}$. 
Step 3 - Thanks to assumption 1.2 and as in Proof of Lemma 4.1, Step 2, we then extend $\beta_{2}$ into $\Omega_{0}$, so that

- $\beta_{2 \mid \Omega_{k}} \in \mathcal{C}^{2}\left(\overline{\Omega_{k}}\right)$ and $\beta_{2}(x)>0$ for any $x \in \bar{\Omega}$;

- $\left[\beta_{2}\right]=0$ and $\left[a \partial_{n} \beta_{2}\right]=0$ on $S$.

- $\beta_{2 \mid \Gamma}$ is constant and $\partial_{n} \beta_{2}(x)<0$ for any $x \in \Gamma$.

- There exists $r>0$ such that $\left|\nabla \beta_{2}\right| \geq r$ in $\Omega \backslash \omega$.

Note that $\left(a \partial_{n} \beta_{2}\right)(x)=-g^{\prime}(x)<0$ for any $x \in \overline{S^{+}}$.

Step 4 - There exist functions $h \in \mathcal{C}^{2}(S), g \in \mathcal{C}^{1}(S)$ so that:

- $h=2$ on $S^{00}, h=4-\beta_{2}$ in $\overline{S^{+}} \cup V$;

- $h(x)<\beta_{2}(x)$ for all $x \in S^{+}, h(x)>\beta_{2}(x)$ for all $x \in S^{-}, h(x)>0$ for all $x \in S$;

- $g=\rho^{-\frac{1}{2}}\left|\nabla^{\prime} h\right|=\rho^{-\frac{1}{2}}\left|\nabla^{\prime} \beta_{2}\right|$ in $V$ and $g(x)>0$ for any $x \in S$.

Step 5 - Following Step 5 of the proof of lemma 2.1, there exist $r>0$ and a function $\beta_{1} \in \mathcal{C}^{2}\left(\overline{\Omega_{0}}\right)$ so that

- $\beta_{1 \mid S}=h$ and $\partial_{n} \beta_{1}=\frac{1}{a_{0}} g$ on $S$;

- $\beta_{1 \mid \Gamma}$ is constant and $\partial_{n} \beta_{1}(x)<0$ for all $x \in \Gamma$;

- $\beta_{1}(x)>0$ for any $x \in \overline{\Omega_{0}}$;

- $\left|\nabla \beta_{1}\right|(x) \geq r>0$ for any $x \in \overline{\Omega_{0}} \backslash \omega$.

Step 6 - We then extend $\beta_{1}$ into $\Omega_{1}$ so that

- $\beta_{1 \mid \Omega_{1}} \in \mathcal{C}^{2}\left(\overline{\Omega_{1}}\right)$, and $\beta_{1}(x)>0$ for any $x \in \overline{\Omega_{1}}$,

- $\left[\beta_{1}\right]=0$ and $\left[a \partial_{n} \beta_{1}\right]=0$ on $S$,

- $\left|\nabla \beta_{1}\right| \geq r^{\prime}>0$ in $\Omega_{1} \backslash B$,

- $\beta_{1 \mid \mathcal{O}}=\frac{1}{2} \min \beta_{2 \mid \overline{\Omega_{1}}}>0$ and $\partial_{n} \beta_{1}(x)>0$ for all $x \in \mathcal{O}$.

(We follow [3, Steps 1, 2 of the Proof of Lemma 3.2] for the construction of $\beta_{1}$ near $\partial \mathcal{O}$ and steps 5,6 of lemma 2.1 for the extension of $\beta_{1}$ near $S$ ).

Step 7 - As in [3, Step 3, Proof of Lemma 3.2] we can construct $\beta_{1}$ so that $\beta_{1} \leq \frac{1}{2} \beta_{2}$ in $\mathcal{O}$.

\section{References}

[1] J. L. Lions and E. Magenes. Problèmes aux limites non homogènes et applications, volume 1. Dunod, Paris, 1968.

[2] A. Pazy. Semigroups of Linear Operators and Applications to Partial Differential Equations, volume 44. A.M.S, Springer-Verlag, New York, 1984.

[3] A. Doubova, A. Osses, and J.-P. Puel. Exact controllability to trajectories for semilinear heat equations with discontinuous diffusion coefficients. ESAIM COCV, 8:621-661, June 2002. 
[4] O. Poisson. Uniqueness and Hölder stability of discontinuous diffusion coefficients in three related inverse problems for the heat equation. Inverse Problems, to appear, 24, 2008.

[5] A. Fursikov and O. Yu. Imanuvilov. Controllability of evolution equations. Seoul National University, Korea. Lecture Notes, 34, 1996.

[6] V. Barbu. Exact Controllability of the superlinear heat equation. Appl. Math. Optim., 42:73-89, 2000.

[7] A. Doubova, E. Fernández-Cara, M. Gonzales-Burgos, and E. Zuazua. On the controllability of parabolic systems with a nonlinear term involving the state and the gradient. SIAM J. Control Optim., 41:798-819, 2002.

[8] E. Fernández-Cara and E. Zuazua. Null and approximate controllability for weakly blowing up semilinear heat equations. Ann. Inst. H. Poincaré, Analyse non linéaire., 17:583-616, 2000.

[9] A. Benabdallah, Y. Dermenjian, and J. Le Rousseau. On the controllability of linear parabolic equations with an arbitrary control location for a stratified media. C. R. Acad. Sci, Paris, Ser. I, 344:357-362, May 2007.

[10] J. Le Rousseau. Carleman estimate and controllability results for the one - dimensional heat equation with bv coefficients. J. Diff. Equations, 233:417-447, 2007.

[11] J. Le Rousseau and L. Robbiano. Carleman estimate for elliptic operators with coefficients with jumps at an interface in arbitrary dimension and application to the null controllability of linear parabolic equations. CNRS, HAL (on web): hal-00193885, version 1, 2007.

[12] Lars Hormander. The Analysis of Linear Partial Differential Equations. Springer-Verlag, Berlin, 1983. 